\title{
Rifampicin may be repurposed for COVID-19 treatment: Insights from an in-silico study
}

\section{Yamini Pathak}

Gautam Buddha University, Greater Noida, India

Amaresh Mishra

Gautam Buddha University, Greater Noida, India

Vishwas Tripathi ( $\nabla$ drvishwastripathi@gmail.com )

Gautam Buddha university, Greater Noida

\section{Research Article}

Keywords: COVID-19, Drug repurposing, Protease inhibitor, Rifampicin

Posted Date: April 14th, 2020

DOI: https://doi.org/10.21203/rs.3.rs-22546/v1

License: (c) (i) This work is licensed under a Creative Commons Attribution 4.0 International License. Read Full License 


\section{Abstract}

The ongoing Coronavirus disease 2019 (COVID-19) outbreak has led to global epidemics with high morbidity and mortality. However, there are no efficacious therapeutic options available for the treatment of COVID-19. Considering this medical emergency, drug repurposing is the need of the hour. Therefore, this study aimed to determine possible COVID-19 drug candidates using virtual screening of selected FDA approved drugs by targeting the main protease (Mpro) of novel coronavirus (SARS-CoV-2). In this study, first, we have done an extensive literature search to find out the marketed FDA approved drug compounds available for human therapeutic applications having anti-viral and anti-bacterial properties. The preliminary reports paved the way for further studies that revealed some of the drugs approved to treat other viral or bacterial infections may be a promising drug candidate and can be repurposed for the treatment of COVID-19. Using a computer-aided drug designing approach, novel candidates for drug repurposing were identified. Based on our preliminary screening supported by previous works of literature, we selected Rifampicin, Ciclesonide, Reserpine, Loperamide, Elvitegravir, Brivudine, Pentoxifylline, Eugenol, Isoniazid, Tinidazole, Diethylcarbamazine, and Vancomycin to target the SARS-CoV-2 main protease (Mpro). These compounds were examined based on docking studies using Autodock 4.2 Autodock 4.2., visualization of the docked results using Pymol version 1.7.4.5 Edu and prediction of two main toxicity aspects hepatotoxicity and cytotoxicity to check the safety of the hit compounds. Selected drugs were ranked for potential efficacy against COVID-19 based on their binding energy. The docking results were found in the order of Rifampicin (-11.16), Ciclesonide (-8.77), Reserpine (-7.76), Loperamide (-7.63), Elvitegravir (-6.58), Brivudine (-6.06), Pentoxifylline (-5.64), Eugenol (-4.78), Isoniazid (-4.71), Tinidazole (-4.51), Diethylcarbamazine (-4.66), Vancomycin (-4.11). ). In the current study, Rifampicin appeared as the most promising drug showing a very good binding energy. Based on our preliminary insights from in silico studies, we propose that Rifampicin may be repurposed for the treatment of COVID19 however it is pertinent to mention here that our findings need further validation by in vitro studies and clinical trials. In summary, the findings of this study suggest Rifampicin might be a promising drug for targeting the COVID-19 protease.

\section{Introduction}

A newly identified coronavirus strain (or severe acute respiratory syndrome, SARS-CoV-2) was reported in Wuhan, China, in late December 2019 (Paraskevis et al. 2020). As of 8 April 2020, a total of 1,478,469 cases of COVID-19 were confirmed in the world including 86,748 deaths. The disease caused by noble coronavirus was named as the coronavirus disease 2019 (COVID-19) by the World Health Organization (WHO) released on 11 February 2020 (World Health Organization (WHO) 2020). According to the current situation, this pandemic is still ongoing, lacking efficacious therapeutic options. However, the steps taken to reduce the severity of infection remain limited to supportive strategies intended to avoid further complications of coronavirus infection (Zhang et al. 2020).

Now researchers are racing against time to discover an effective drug candidate by targeting protease inhibitors to block the SARS-CoV-2 main protease (Mpro). The molecular structure of COVID-19 main 
protease $\left(\mathrm{M}^{\text {pro }}\right)$ as determined by Liu et al. (2020) represents a potential target for the inhibition of coronavirus replication (Xu et al. 2020). Some retrospective findings of past outbreaks have helped to examine possible combinations like the protease inhibitor lopinavir/ritonavir, commonly used in the treatment of HIV patients ( $L u$. 2020). Taken together, these findings indicate that protease inhibitors may block the main protease of the novel coronavirus and this could be an effective approach in checking the virus replication cycle (Sheahan et al. 2020). The therapeutic strategies have been focused to identify the viral protease inhibitor that does not inhibit important human protease activity.

Drug repurposing (also known as drug repositioning or reprofiling) is a technique to identify new applications for certified or investigational drugs outside the original medical indication. Thus, in this study, using in silico approach, several FDA approved drugs that exhibit the potential for drug repurposing, e.g., Brivudine, Ciclesonide, Diethylcarbamazine, Elvitegravir, Eugenol, Isoniazid, Loperamide, Lopinavir, Pentoxifylline, Reserpine, Rifampicin, Ritonavir, Tinidazole, Vancomycin (https://www.drugbank.ca/) have been virtually screened for prediction of the potential drug candidate and binding affinity against coronavirus main protease $\left(\mathrm{M}^{\mathrm{pro}}\right)$. Therefore, great attention has been paid to the drug repurposing approach (Talevi and Bellera 2020). The initially conceived technique of using proven medications to treat other symptoms for one disorder has found success across medical fields. Such repurposing medication promises to provide patients with quicker access to drugs while reducing costs in the long and difficult drug development cycle (Parvathaneni et al. 2019). Therefore, the current study aimed to elucidate the Novel Drug Repurposing Strategy to investigate the candidate drugs for in vitro studies, in vivo studies and clinical trials and to attain mechanistic insights into the outcomes of such research studies. In this study, we employed in silico methods to investigate novel protease inhibitor for the suppression of the antiviral effect of coronavirus in order to target SARS-CoV-2 main protease $\left(\mathrm{M}^{\mathrm{pro}}\right)$.

\section{Materials \& Methods}

2.1 Proteins/Macromolecules: The crystal structure of COVID-19 main protease (M ${ }^{\text {pro }}$ ) (PDB ID: 6LU7) structures were retrieved from PDB (https://www.rcsb.org/), in .pdb format. PDB is an archive for the crystal structures of biological macromolecules, worldwide. The structures of the target protein SARSCoV-2 $\mathrm{M}^{\text {pro }}$ with a covalent inhibitor, N3, was available in the PDB and it contains two chains, A and B, which form a homodimer. Thereafter the chain A of PDB file was optimized for macromolecule preparation.

2.2 Literature search and drug selection: The list and structures of FDA approved drugs were obtained from the drug bank (https://www.drugbank.ca/). The 12 FDA-approved drug compounds (Table 1) used in the present study were Brivudine, Ciclesonide, Diethylcarbamazine, Elvitegravir, Eugenol, Isoniazid, Loperamide, Lopinavir, Pentoxifylline, Reserpine, Rifampicin, Ritonavir, Tinidazole, Vancomycin against viral protease that could block SARS-CoV-2 protease. Thereafter, the molecules which were optimized, saved in the PDB format for further processing. 
Table 1: List of FDA-approved (marketed) drug compounds

\begin{tabular}{|c|c|}
\hline Drug Bank ID & Drug name \\
\hline DB03312 & Brivudine \\
\hline DB01410 & Ciclesonide \\
\hline DB00711 & Diethylcarbamazine \\
\hline DB09101 & Elvitegravir \\
\hline DB09086 & Eugenol \\
\hline DB00951 & Isoniazid \\
\hline DB00836 & Loperamide \\
\hline DB01601 & Lopinavir \\
\hline DB00806 & Pentoxifylline \\
\hline DB00206 & Reserpine \\
\hline DB01045 & Rifampicin \\
\hline DB00503 & Ritonavir \\
\hline DB00911 & Tinidazole \\
\hline DB00512 & Vancomycin \\
\hline
\end{tabular}

2.3 Toxicity analysis: Toxicity analysis of selected FDA approved market available drug compounds was done by the ProTox-II http://tox.charite.de/protox_II/ web server. ProTox-II analyses and predicts various toxicity parameters like organ toxicity, cytotoxicity etc,. and association of the selected molecule with various adverse pathways, if, any.

2.4 Determination of Active Sites: Active sites were determined by the binding pocket analysis using CASTp 3.0 software (http://sts.bioe.uic.edu/castp/ server). The active site residues were used to evaluate the Grid box and docking results. The binding pocket of the ligand in the catalytic site was manually obtained followed by verification by docking analysis.

2.5 Molecular Docking: Molecular docking analysis was done using a local search algorithm to investigate the most preferred binding mode of the selected FDA approved drugs. The binding affinity of the ligand with the active site of the enzyme was determined by energy score (S, Kcal/mol), low energy score indicates good affinity. Molecular docking was performed using the crystal structure of COVID-19 main protease $\mathrm{M}^{\text {pro }}$ using Autodock 4.2. (Pymol version 1.7.4.5 Edu) were utilized for visualization of the docked results.

\section{Results}

\subsection{Toxicity evaluation of FDA approved drugs involved in the study}

The individual toxicities of FDA approved (marketed) drugs were predicted by using ProTox-II. The toxicity analysis was performed in order to predict the safety aspects of the FDA approved drug. The major toxicity endpoints were taken into consideration and the drugs which were not following the safety 
parameters of toxicity endpoints were not taken for further analysis in our priority list. As shown in Table 2, ProTox-II toxicity prediction software gave result mainly associated with three main toxicity aspects cytotoxicity, carcinogenicity and mutagenicity to check the safety aspects of the FDA approved market available drug compounds. According to the toxicological data, none of the selected FDA approved market available drug compound was shown potential cytotoxicity, carcinogenicity, and mutagenicity.

Table 2: Toxicity predictions for selected FDA approved (marketed) drug compounds

\begin{tabular}{|c|c|c|c|c|c|c|}
\hline S.No. & Compounds & Toxicity class & LD50 (mg/kg) & Cytotoxicity & Carcinogenicity & Mutagenicity \\
\hline $\mathbf{1 .}$ & Ritonavir* & 4 & 1000 & Inactive & Inactive & Inactive \\
\hline $\mathbf{2 .}$ & Lopinavir* & 5 & 5000 & Inactive & Inactive & Inactive \\
\hline $\mathbf{3 .}$ & Rifampicin & 4 & 500 & Inactive & Inactive & Inactive \\
\hline $\mathbf{4 .}$ & Ciclesonide & 4 & 2000 & Inactive & Active & Inactive \\
\hline $\mathbf{5 .}$ & Reserpine & 2 & 50 & Active & Active & Inactive \\
\hline $\mathbf{6 .}$ & Loperamide & 4 & 1190 & Inactive & Inactive & Inactive \\
\hline $\mathbf{7 .}$ & Elvitegravir & 4 & 800 & Inactive & Inactive & Inactive \\
\hline $\mathbf{8 .}$ & Brivudine & 6 & 8400 & Inactive & Inactive & Inactive \\
\hline $\mathbf{9 .}$ & Pentoxifylline & 4 & 780 & Inactive & Inactive & Inactive \\
\hline $\mathbf{1 0 .}$ & Eugenol & 4 & 1930 & Inactive & Inactive & Inactive \\
\hline $\mathbf{1 1 .}$ & Isoniazid & 3 & 133 & Inactive & Active & Inactive \\
\hline $\mathbf{1 2 .}$ & Diethylcarbamazine & 4 & 660 & Inactive & Inactive & Inactive \\
\hline $\mathbf{1 3 .}$ & Tinidazole & 5 & 2710 & Inactive & Active & Active \\
\hline $\mathbf{1 4 .}$ & Vancomycin & 5 & 5000 & Inactive & Inactive & Inactive \\
\hline
\end{tabular}

3.2 Binding Site Prediction: The structures and amino acid residues found in the active site pockets of 6 LU7 were visualized in figure 1. Both area and volume shown are (Richards') solvent-accessible surface area/volume.

Table 3: The structure and binding pockets of the SARS-CoV-2 protease (PDB ID: 6lu7) 


\begin{tabular}{|c|c|c|c|c|}
\hline No. & $\begin{array}{c}\text { PDB } \\
\text { ID }\end{array}$ & Macromolecule & Native Ligand & Active site \\
\hline 1. & 6LU7 & & & $\begin{array}{l}\text { THR24,THR25,THR26,LEU27,HIS41,CYS44,THR45, } \\
\text { SER46,MET49,PRO52,TYR54,PHE140,LEU141, } \\
\text { ASN142,GLY143,SER144,CYS145,HIS163,HIS164, } \\
\text { MET165,GLU166,LEU167,PRO168,HIS172,ASP187, } \\
\text { ARG188,GLN189,THR190,GLN192 }\end{array}$ \\
\hline
\end{tabular}

3.3 Docking analysis: All the 12 compounds and the standard reference compounds were further docked by Autodock 4.2. The 12 compounds obtained from drug bank were screened for molecular docking. Among them, 4 compounds approved by the FDA have a docking score better than $-7.0 \mathrm{kcal} / \mathrm{mol}$. Thus among 12 compounds and the standard reference compounds, 4 hits were found to have good affinities in terms of docking scores. The results of our docking study revealed that among selected drugs, interestingly, two drugs Rifampicin and Ciclesonide showed the best affinity even better than the reference compounds Ritonavir and Lopinavir.

Table 4: Molecular docking analysis of several compounds against COVID-19 main protease (M ${ }^{\text {pro }}$ ) (PDB ID: 6LU7) *Standard Compounds

\begin{tabular}{|c|c|c|c|}
\hline Drug name & Protein & Affinity (kcal/mol) & Chain \\
\hline Ritonavir* & \multirow{14}{*}{$\begin{array}{c}\text { COVID-19 main protease } M^{\text {pro }} \\
\text { (PDB ID: 6LU7) }\end{array}$} & -8.52 & A \\
\hline Lopinavir* & & -8.5 & A \\
\hline Rifampicin & & -11.16 & A \\
\hline Ciclesonide & & -8.77 & A \\
\hline Reserpine & & -7.76 & A \\
\hline Loperamide & & -7.63 & A \\
\hline Elvitegravir & & -6.58 & A \\
\hline Brivudine & & -6.06 & A \\
\hline Pentoxifylline & & -5.64 & A \\
\hline Eugenol & & -4.78 & A \\
\hline Isoniazid & & -4.71 & A \\
\hline Diethylcarbamazine & & -4.66 & A \\
\hline Tinidazole & & -4.51 & A \\
\hline Vancomycin & & -4.11 & A \\
\hline
\end{tabular}

\section{Discussion}

The outbreak of COVID-19 has been declared a global pandemic by the WHO, and the development of effective treatment for COVID-19 is in an urgent need (World Health Organization 2020). COVID-19 main protease $\mathrm{M}^{\text {pro }}$ is responsible for proteolytic processing, viral replication, and the establishment of the 
infection cycle. In a recent study by Liu et al. (2020), the crystal structure of COVID-19 main protease $\mathrm{M}^{\text {pro }}$ has been reported (PDB ID: 6LU7) (Jin et al. 2020) and this may significantly facilitate the discovery of potential drug compounds to prevent COVID-19 infection by targeting its main protease $\mathrm{M}^{\text {pro }}$ through existing drug libraries for drug repurposing. Lopinavir and ritonavir are well-established protease inhibiting drugs for HIV(Sheean et al. 2008). In several studies, both drugs were also proposed to treat SARS and the Middle East respiratory syndrome (MERS)(Chu et al. 2004). This combination has also been used in COVID-19 patients in order to control COVID-19 infection (Lim et al. 2020). Therefore, in this study, we have taken these drugs as a standard reference to compare the efficacy of the binding of our selected FDA approved drugs. After identification of the active sites of COVID-19 main protease $\mathrm{M}^{\text {pro }}$ (PDB: 6LU7), we further performed docking study of our selected compounds Rifampicin, Ciclesonide, Reserpine, Loperamide, Elvitegravir), Brivudine, Pentoxifylline, Eugenol, Isoniazid, Tinidazole, Diethylcarbamazine, and Vancomycin as potential inhibitors of the COVID-19 main protease $\mathrm{M}^{\text {pro }}$. The binding energies obtained from docking 6LU7 with selected FDA approved drugs showed inhibition potential of these drugs in the order of, ranked by affinity $(\Delta G)$ i.e. Rifampicin, Ciclesonide, Reserpine, Loperamide, Elvitegravir, Brivudine, Pentoxifylline, Eugenol, Isoniazid, Tinidazole, Diethylcarbamazine, and Vancomycin was $-11.16,-8.77,-7.76,-7.63,-6.58,-6.06,-5.64,-4.78,-4.71,-4.51,-4.66$, and $-4.11 \mathrm{kcal} / \mathrm{mol}$ respectively. One of the very interesting findings of our study is that among the selected FDA approved drugs, two drugs Rifampicin and Ciclesonide were giving binding affinity even better than the reference drugs. To sum up, our in silico preliminary findings suggest that Rifampicin may be used as a repurposed drug for the treatment of COVID-19. However, these findings should further be validated at the in vitro and clinical trials.

\section{Conclusion}

Drug repurposing is an attractive option for life-threatening pandemics like COVID-19 for the rapid identification of potential therapeutics. This study aimed to examine several FDA approved drug compounds that could be used as repurposed drugs to inhibit COVID-19 infection. In this in silico study, Rifampicin was identified as the best hit among the selected drugs. Interestingly, the binding affinity of Rifampicin was found to be much better than the selected reference drugs as per docking results. Further, the major toxicity end pint prediction results were also good for rifampicin. Therefore the results of this study indicate that Rifampicin, a well-established medicine for the treatment of tuberculosis has a stronger binding affinity for COVID-19 main protease Mpro in comparison to the other drug compounds taken in this study. To sum up, our preliminary in silico findings suggest that Rifampicin may be used as a repurposed drug for the treatment of COVID-19. However, it is pertinent to mention here that these findings warrant further in vitro and clinical trials in order to precisely conclude our findings.

\section{References}

1. Chu, C. M., V. C.C. Cheng, I. F.N. Hung, M. M.L. Wong, K. H. Chan, K. S. Chan, R. Y.T. Kao, et al. 2004. "Role of Lopinavir/Ritonavir in the Treatment of SARS: Initial Virological and Clinical Findings." 
Thorax 59 (3): 252-56. https://doi.org/10.1136/thorax.2003.012658.

2. Fahmi, Ismail. 2020. “\#Covid19 Coronavirus Disease 2019.” DroneEmprit 2019 (March): 1-19. https://pers.droneemprit.id/covid19/.

3. Jin, Zhenming, Xiaoyu Du, Yechun Xu, Yongqiang Deng, Meiqin Liu, Yao Zhao, Bing Zhang, et al. 2020. "Structure of Mpro from COVID-19 Virus and Discovery of Its Inhibitors." BioRxiv. https://doi.org/10.1101/2020.02.26.964882.

4. Lim, Jaegyun, Seunghyun Jeon, Hyun Young Shin, Moon Jung Kim, Yu Min Seong, Wang Jun Lee, Kang Won Choe, Yu Min Kang, Baeckseung Lee, and Sang Joon Park. 2020. “The Author's Response: Case of the Index Patient Who Caused Tertiary Transmission of Coronavirus Disease 2019 in Korea: The Application of Lopinavir/Ritonavir for the Treatment of COVID-19 Pneumonia Monitored by Quantitative RT-PCR." Journal of Korean Medical Science 35 (7): 1-6. https://doi.org/10.3346/jkms.2020.35.e89.

5. Lu, Hongzhou. 2020. "Drug Treatment Options for the 2019-New Coronavirus (2019-NCoV)." BioScience Trends. https://doi.org/10.5582/bst.2020.01020.

6. Paraskevis, D., E. G. Kostaki, G. Magiorkinis, G. Panayiotakopoulos, G. Sourvinos, and S. Tsiodras. 2020. "Full-Genome Evolutionary Analysis of the Novel Corona Virus (2019-NCoV) Rejects the Hypothesis of Emergence as a Result of a Recent Recombination Event." Infection, Genetics and Evolution. https://doi.org/10.1016/j.meegid.2020.104212.

7. Parvathaneni, Vineela, Nishant S. Kulkarni, Aaron Muth, and Vivek Gupta. 2019. "Drug Repurposing: A Promising Tool to Accelerate the Drug Discovery Process." Drug Discovery Today 24 (10): 207685. https://doi.org/10.1016/j.drudis.2019.06.014.

8. Sheahan, Timothy P., Amy C. Sims, Sarah R. Leist, Alexandra Schäfer, John Won, Ariane J. Brown, Stephanie A. Montgomery, et al. 2020. "Comparative Therapeutic Efficacy of Remdesivir and Combination Lopinavir, Ritonavir, and Interferon Beta against MERS-CoV." Nature Communications. https://doi.org/10.1038/s41467-019-13940-6.

9. Sheahan, Timothy P., Amy C. Sims, Sarah R. Leist, Alexandra Schäfer, John Won, Ariane J. Brown, Stephanie A. Montgomery, et al. 2020. "Comparative Therapeutic Efficacy of Remdesivir and Combination Lopinavir, Ritonavir, and Interferon Beta against MERS-CoV." Nature Communications. https://doi.org/10.1038/s41467-019-13940-6.

10. Sheean et al., 2013. 2008. "वास्तNIH Public Access." Bone 23 (1): 1-7. https://doi.org/10.1038/jid.2014.371.

11. World Health Organization. 2020. "WHO Director-General's Opening Remarks at the Mission Briefing on COVID-19." Https://Www.Who.Int/Dg/Speeches/Detail/Who-Director-General-s-Opening-Remarksat-the-Media-Briefing-on-Covid-19--11-March-2020. 2020. https://doi.org/11 March 2020.

12. World Health Organization (WHO). 2020. "Novel Coronavirus (2019-NCoV)." WHO Bulletin.

13. WHO (World Health Organization). 2020. "Statement on the Meeting of the International Health Regulations (2005) Emergency Committee Regarding the Outbreak of Novel Coronavirus (2019NCoV)." WHO Newsletter, 2020. 
14. Xu, Zhijian, Cheng Peng, Yulong Shi, Zhengdan Zhu, Kaijie Mu, Xiaoyu Wang, and Weiliang Zhu. 2020. "Nelfinavir Was Predicted to Be a Potential Inhibitor of 2019-NCov Main Protease by an Integrative Approach Combining Homology Modelling, Molecular Docking and Binding Free Energy Calculation." BioRxiv. https://doi.org/10.1101/2020.01.27.921627.

15. Zhang, Deng hai, Kun lun Wu, Xue Zhang, Sheng qiong Deng, and Bin Peng. 2020. "In Silico Screening of Chinese Herbal Medicines with the Potential to Directly Inhibit 2019 Novel Coronavirus." Journal of Integrative Medicine 18 (2): 152-58. https://doi.org/10.1016/j.joim.2020.02.005.

\section{Figures}

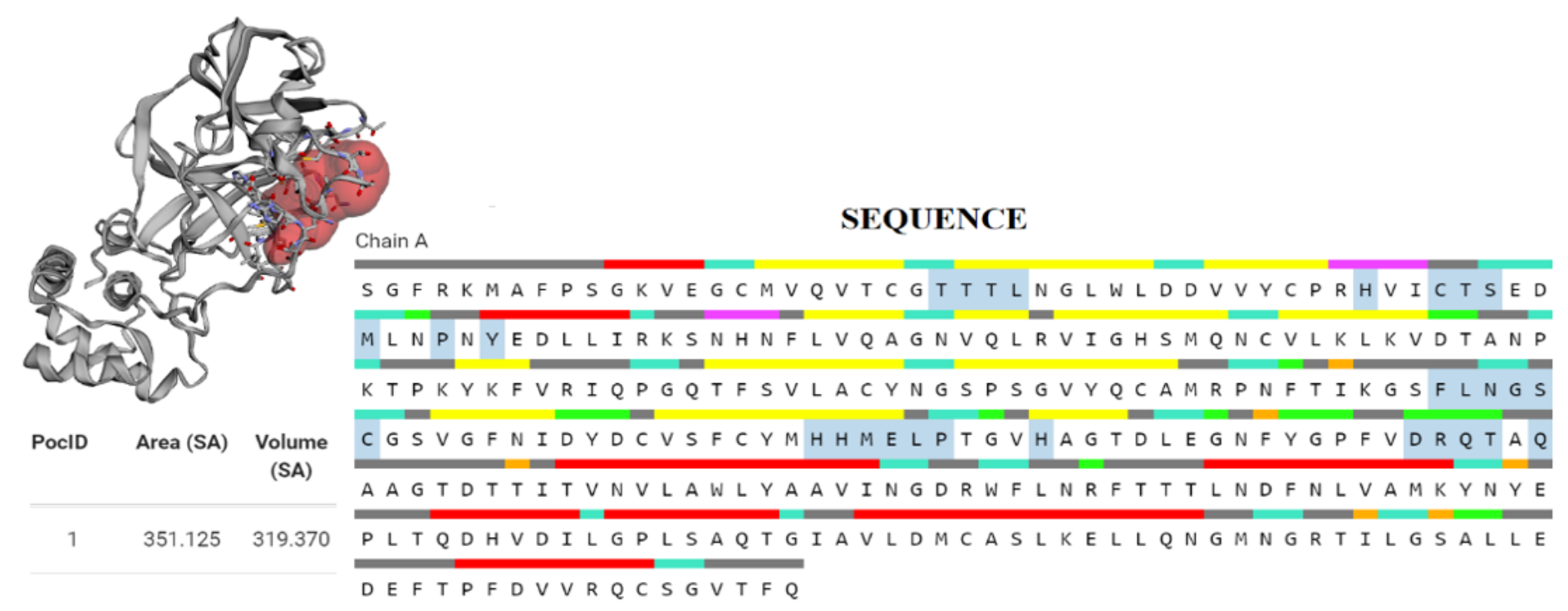

Figure 1

Sequence and active site pockets of SARS-CoV-2 protease (PDB ID: 6lu7) retrieved from Castp 


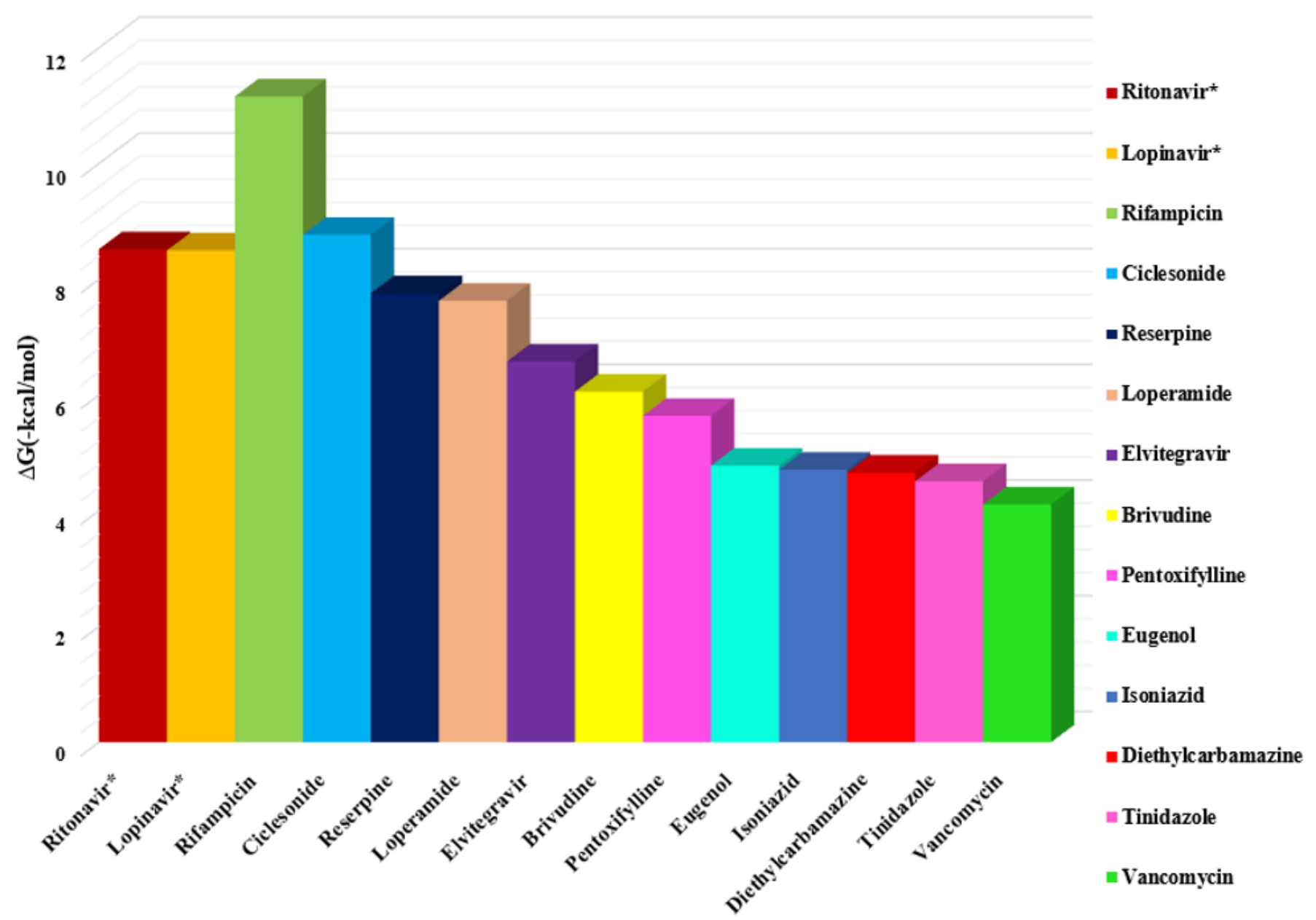

Compounds

Figure 2

Histogram showing molecular docking results between COVID-19 main protease Mpro (PDB ID: 6LU7) and several drug compounds (the binding energy value $\Delta \mathrm{G}$ is shown in minus $\mathrm{kcal} / \mathrm{mol}$ ). * Standard compounds 


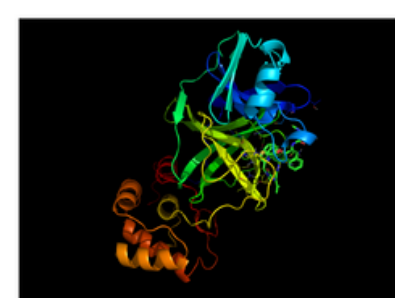

A. Ritonavir

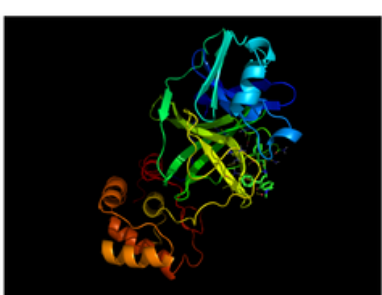

F. Loperamide

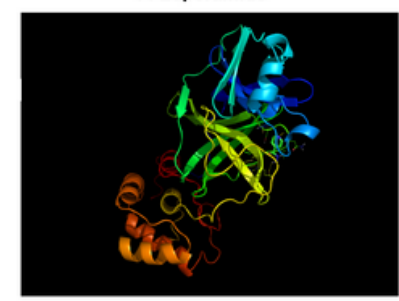

K. Isoniazid

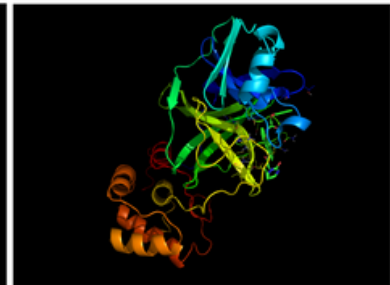

B. Lopinavir

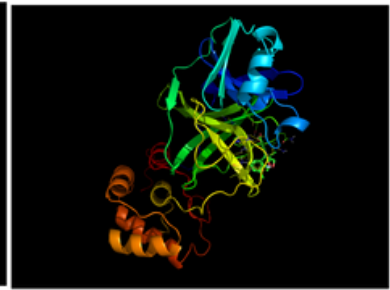

G. Elvitegravir

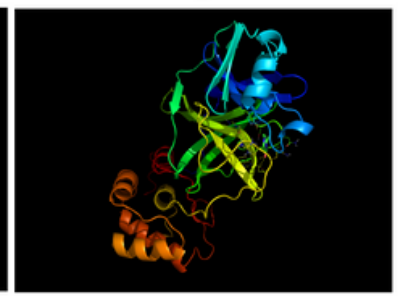

L. Diethylcarbamazine

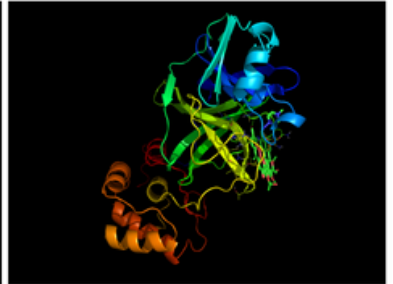

C. Rifampicin

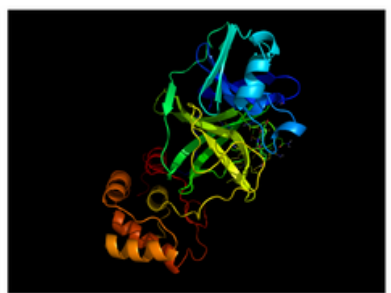

H. Brivudine

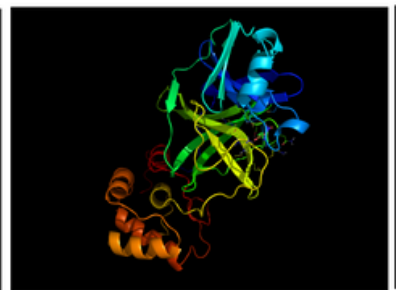

M. Tinidazole

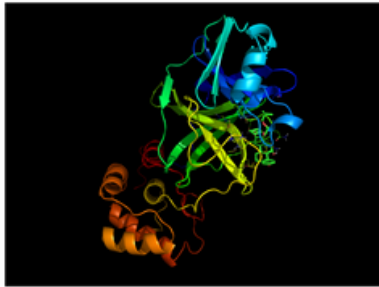

D. Ciclesonide

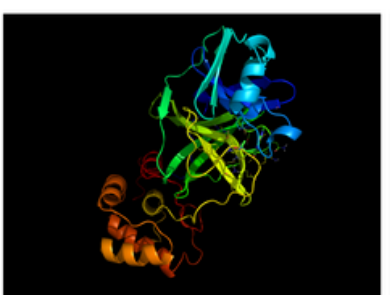

I. Pentoxifylline

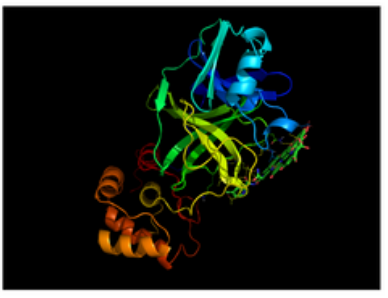

N. Vancomycin

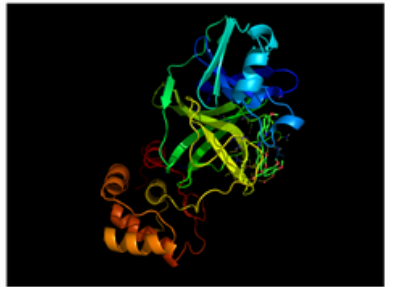

E. Reserpine

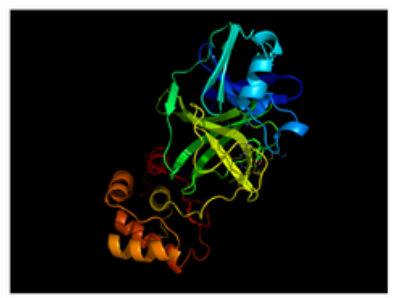

J. Eugenol

\section{Figure 3}

Docking analysis visualisation of COVID-19 main protease (Mpro) (PDB ID: 6LU7) binding with Ritonavir, Lopinavir, Rifampicin, Ciclesonide, Reserpine, Loperamide, Elvitegravir, Brivudine, Pentoxifylline, Eugenol, Isoniazid, Diethylcarbamazine, Tinidazole, Vancomycin using Pymol. 

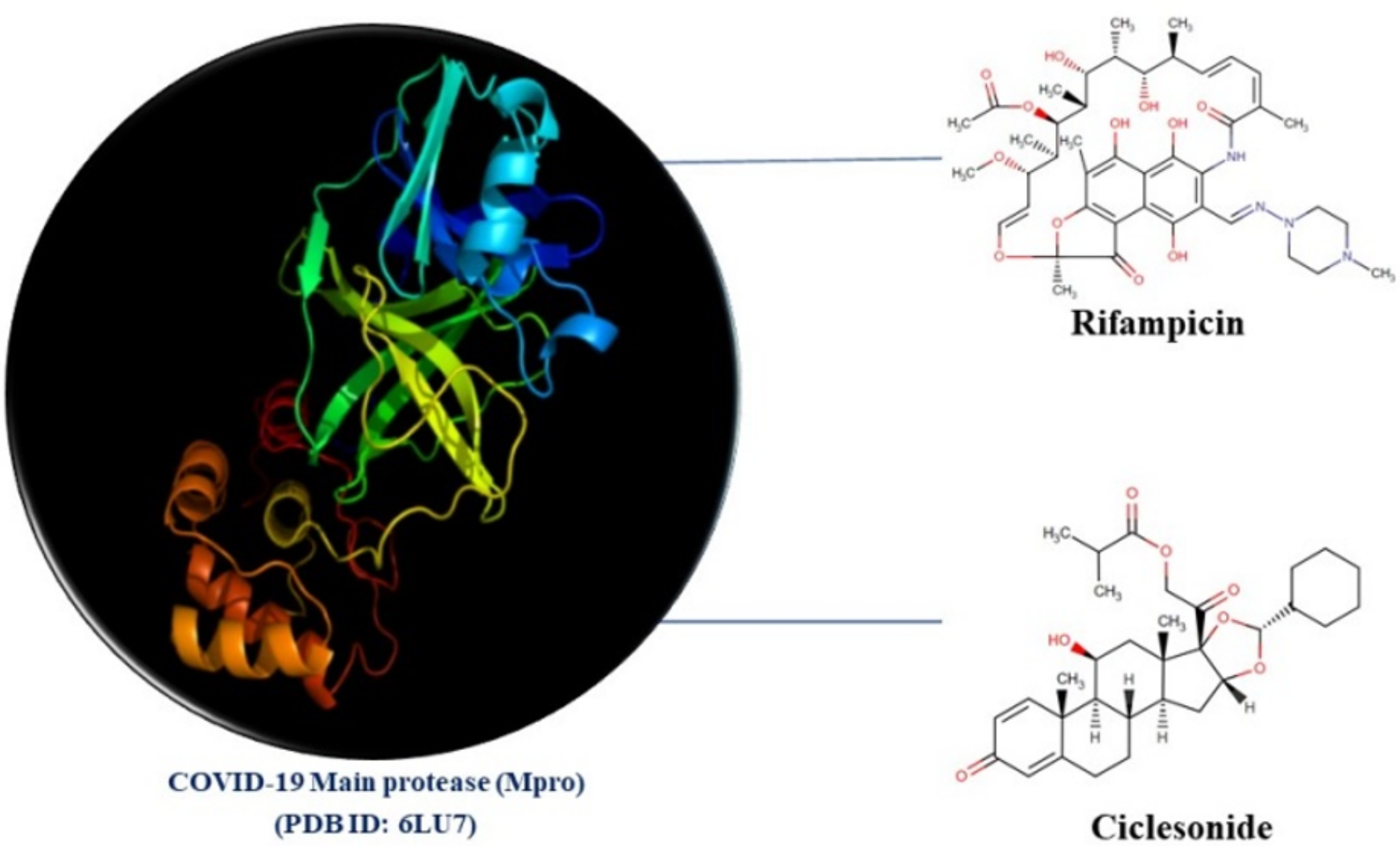

Ciclesonide

Figure 4

FDA approved drugs having a docking score better than lopinavir/ritonavir (more than $-8.0 \mathrm{kcal} / \mathrm{mol}$ ) against COVID-19 main protease (Mpro) (PDB ID: 6LU7). 


\section{Retrieval of PDB ID : 6LU7 and preparation of proteins and ligands}

Compound Source= Drug Bank https://www.drugbank.ca/

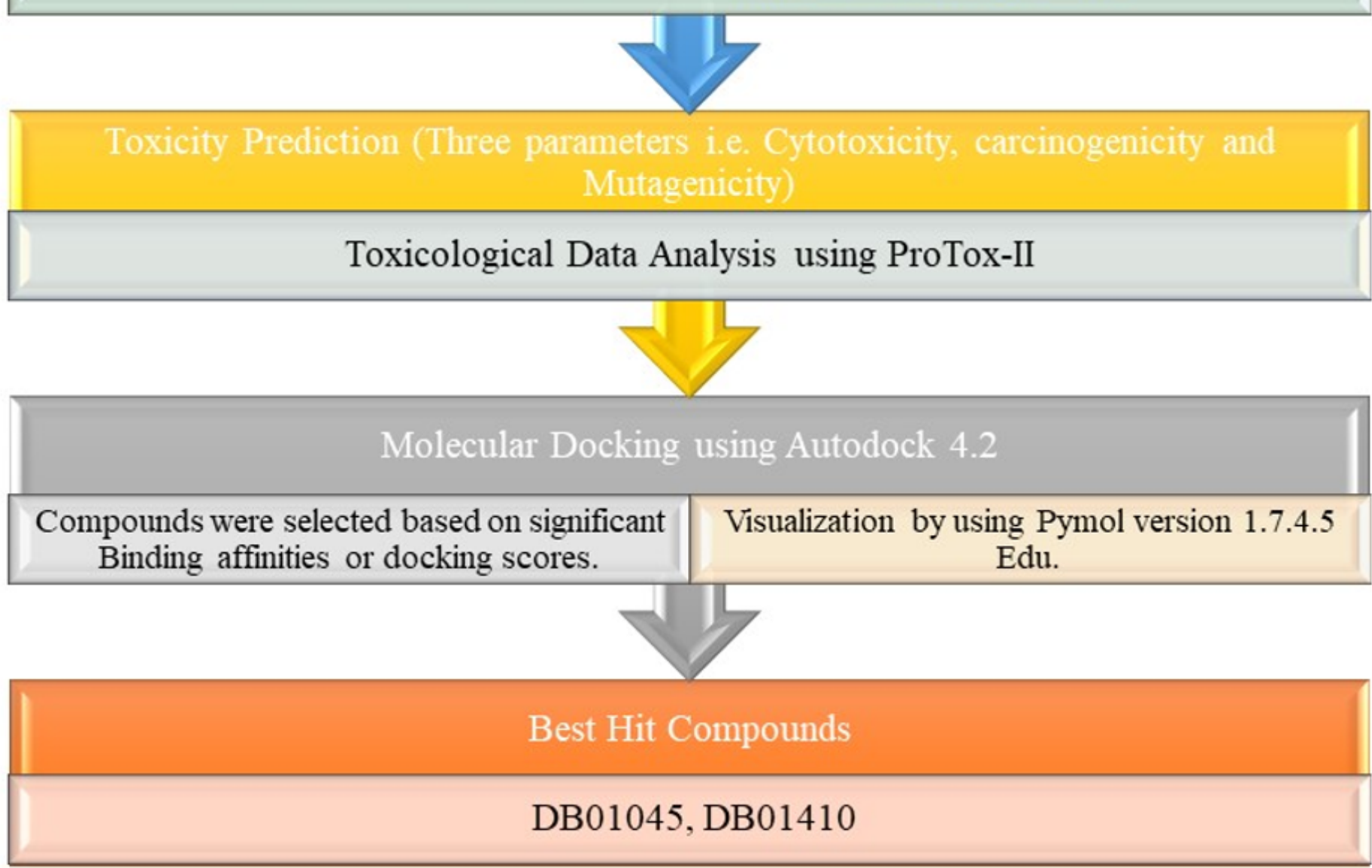

Figure 5

Schematic representation of the overall workflow of Toxicity prediction, sequential virtual screening 